\title{
TENSOR PRODUCTS OF MODULES, RIGIDITY AND LOCAL COHOMOLOGY
}

\author{
CRAIG HUNEKE and ROGER WIEGAND
}

Dedicated to the memory of Maurice Auslander

In this paper we continue our study of the rigidity of Tor over hypersurfaces and regular rings, begun in [HW]. A starting point of this paper is a result in a paper of Luk and Yau [LY] which gives a cohomological criterion for a vector bundle over complex projective space to be a direct sum of line bundles. A commutative algebra interpretation of this result gives a condition, over a regular local ring $R$, for a reflexive module $M$ to be free. Basically the condition amounts to saying that a second local cohomology module vanishes, namely $H_{\mathbf{m}}^{2}\left(M \otimes_{R} M^{*}\right)=0$. It is known that a torsion-free module $M$ over an integrally closed domain is free iff $M \otimes_{R} M^{*}$ is reflexive. One possible interpretation of the result of Luk and Yau is to speculate that there is a cohomological rigidity theorem which says if one local cohomology module of a tensor product vanishes, then all lower ones vanish. Under suitable assumptions, we are able to prove this. Since the vanishing of the first $r$ local cohomology modules of $N$ is equivalent to $\operatorname{depth}(N)>r$, such a rigidity theorem is really a statement about the depth of the tensor product of two modules, provided a single local cohomology module of the tensor product vanishes. Theorem 2.4 states:

2.4. THEOREM Let $M$ and $N$ be finitely generated nonzero modules over a local ring $(R, \mathbf{m})$ of dimension $d$ whose completion is the quotient of an unramified (or equicharacteristic) regular local ring by a nonzero element. Let $r$ be an integer with $0 \leq r<d$. Assume that $M \otimes_{R} N$ satisfies Serre's condition $\left(\mathrm{S}_{\mathrm{r}+1}\right)$ on the punctured spectrum of $R$. Further assume that $N$ has finite projective dimension. The following conditions are equivalent:

(1) $\mathrm{H}_{\mathrm{m}}^{r}\left(M \otimes_{R} N\right)=0$ and both $M$ and $N$ have depth at least $r$.

(2) $\operatorname{depth}(M)+\operatorname{depth}(N) \geq d+r+1$.

Both authors were partialy supported by grants from the National Science Foundation.

Received November 6, 1995. 
(3) $\operatorname{depth}\left(M \otimes_{R} N\right) \geq r+1$.

(4) $M \otimes_{R} N$ satisfies Serre's condition $\left(\mathrm{S}_{\mathrm{r}+1}\right)$.

Thus, not only is a rigidity theorem true, but we are able to give necessary and sufficient conditions in terms of the depth of the modules for the tensor product to have high depth. Moreover, the theorem is true for hypersurfaces, provided one of the modules has finite projective dimension. In Theorem 2.5 we give a rigidity theorem for hypersurfaces without any assumption of finite projective dimension, but we must assume two consecutive local cohomology modules vanish. The theorem of Luk and Yau follows from the special case in which $R=\mathrm{C}\left[X_{0}, \ldots, X_{n}\right]_{\left(X_{0}, \ldots, X_{n}\right)}, r=2, N=M^{*}$, and $M$ is a graded reflexive $R$-module which is free on the punctured spectrum of $R$.

Theorem 2.4 has many nice consequences. For example in (2.6) we recover the main result of an old paper of Auslander and Goldman. Another corollary (2.7) gives that for nonzero reflexive modules over a regular local ring, $\operatorname{Hom}_{R}(M, N)$ satisfies Serre's condition $\left(\mathrm{S}_{3}\right)$ only if it is isomorphic with $M^{*} \otimes_{R} N$, and all the higher Tors of $M^{*}$ and $N$ are zero. If a direct sum of copies of $M$ maps onto $N$ we can conclude $M$ has a nonzero free summand. Later corollaries give depth estimates and rigidity theorems for tensor products of modules which are free on the punctured spectrum of a regular local ring. Proposition 3.6 shows that if $M$ and $N$ are of this form, $M$ has rank 2, and $\mathrm{H}_{\mathbf{m}}^{2}\left(M \otimes_{R} N\right)=0$, then $M^{* *}$ is free. Corollary 3.8 shows that if the second local cohomology module of the tensor product of at least $\operatorname{dim}(\mathbf{R})-2$ such modules is zero, then the double dual of at least one of them is free.

Section 1 gives basic background material including a discussion of an important formula on the depth of a tensor product, which we call the depth formula. In addition an interesting fact is proved about finitely generated modules $M$ and $N$ over hypersurfaces: one of them has finite projective dimension iff all sufficiently high Tors of $M$ and $N$ are zero. See Theorem 1.9.

Section 2 proves most of the main theorems and gives several consequences. Section 3 applies the main theorems to the depth of tensor products of modules that are locally free on the punctured spectrum of a regular local ring. By an abuse of notation, we call such modules vector bundles.

Section 4 gives applications of the main theorems to tensor products of the form $M \otimes_{R} M^{*}$. This is the case studied by Luk and Yau. It is the torsion of this module which plays a role in ramification theory, as first noted by the pioneering papers of Auslander and Goldman [AG], Auslander and Buchsbaum [AB2], and Auslander [A1,2]. Indeed, the proofs of many of our results come from the work of Auslander and his co-authors in the late 1950's and early 1960's. The techniques developed in these papers and reported on 
in Auslander's address at the International Congress in Stockholm in 1962 [A3] are still amazing today.

In Section 5 we translate our theorems on local rings to theorems about vector bundles over projective space. The theorem of Luk and Yau is one of the corollaries of this translation.

Finally in Section 6 we give a new rigidity theorem for Tors which we discovered while trying to prove Theorem 2.4. Except for one special case the theorem says that over an unramified regular local ring, if $\operatorname{Tor}_{i+1}^{R}(M, N)$ is nonzero and has finite length, then the depth of $\operatorname{Tor}_{i}^{R}(M, N)$ must be 0 .

\section{A depth formula.}

Let $M$ and $N$ be nonzero finitely generated modules over a local ring $(R, \mathbf{m})$. We say that $M$ and $N$ satisfy the depth formula provided

$$
\operatorname{depth}(M)+\operatorname{depth}(N)=\operatorname{depth}(R)+\operatorname{depth}\left(M \otimes_{R} N\right) .
$$

Observe that a necessary condition for the depth formula to hold is that $\operatorname{depth}(M)+\operatorname{depth}(N) \geq \operatorname{depth}(R)$. Auslander and Lichtenbaum [A, $(3,1)]$, [L] showed that $M$ and $N$ satisfy the depth formula if $(R, \mathbf{m})$ is regular and $M \otimes_{R} N$ is torsion-free. The main step in the proof is to show that $\operatorname{Tor}_{i}^{R}(M, N)=0$ for all $i \geq 1$. In fact, if $(R, \mathbf{m})$ is a complete intersection the depth formula holds [HW, (2.5)] whenever $\operatorname{Tor}_{i}^{R}(M, N)=0$ for all $i \geq 1$. We record the following observation, pointed out to us by David Jorgensen:

1.2. Lemma. Let $(R, \mathrm{~m})$ be a complete intersection, and let $M$ and $N$ be $R$ modules such that $\operatorname{Tor}_{i}^{R}(M, N)=0$ for all $i>>$. Then $\operatorname{Tor}_{i}^{R}(M, N)=0$ for all $i>\operatorname{dim}(R)$.

Proof. We proceed by induction on the codimension $r$ of $R$, the case $r=0$ (that is, $R$ regular) being clear. Assuming $r>0$, write $R=S /(f)$, where $S$ is a complete intersection of codimension $r-1$. There is a changeof-rings spectral sequence yielding a long exact sequence [L], [HW, (2.1)]

$$
\cdots \rightarrow \mathrm{T}_{i}^{R} \rightarrow \mathrm{T}_{i+1}^{S} \rightarrow \mathrm{T}_{i+1}^{R} \rightarrow \mathrm{T}_{i-1}^{R} \rightarrow \mathrm{T}_{i}^{S} \rightarrow \cdots,
$$

where $\mathrm{T}_{i}^{R}=\operatorname{Tor}_{i}^{R}(M, N)$ and $\mathrm{T}_{i}^{S}=\operatorname{Tor}_{i}^{S}(M, N)$. It follows from (1.2.1) that $\operatorname{Tor}_{i}^{S}(M, N)=0$ for $i>0$, and by induction that $\operatorname{Tor}_{i}^{S}=0$ for $i>\operatorname{dim}(S)=\operatorname{dim}(R)+1$. From (1.2.1) we see that $\mathrm{T}_{i-1}^{R}(M, N)=$ $\operatorname{Tor}_{i+1}^{R}(M, N)$ for all $i \geq \operatorname{dim}(R)+2$. The result follows immediately.

1.3. Proposition. Let $(R, \mathbf{m})$ be a complete intersection, and let $M$ and $N$ be nonzero R-modules satisfying Serre's conditions $\left(\mathrm{S}_{\mathrm{m}}\right)$ and $\left(\mathrm{S}_{\mathrm{n}}\right)$, respectively. If $m+n \geq \operatorname{dim}(R)$ and $\operatorname{Tor}_{i}^{R}(M, N)=0$ for all $i>>0$, then the depth formula (1.1) holds for $M$ and $N$. 
Proof. We may assume that $m+n=d:=\operatorname{dim}(R)$. Since $R$ is Gorenstein, $M$ is an $m$ th syzygy of some $R$-module $X$, and $N$ is an $n$th syzygy of, say, $Y$. (See, e.g., [EG, (3.8)]. Now $\operatorname{Tor}_{i}^{R}(X, Y)=0$ for all $i>>0$, and by the lemma above $\operatorname{Tor}_{i}^{R}(X, Y)=0$ for all $i>d$. The long exact sequence of Tor then implies that $\operatorname{Tor}_{i}^{R}(M, N)=0$ for all $i>0$. By [HW, (2.5)], $M$ and $N$ satisfy the depth formula.

1.4. Corollary. Let $(R, \mathrm{~m})$ be a Noetherian local ring which is a complete intersection. Suppose that $M$ and $N$ are finitely generated $R$-modules which are both free on the punctured spectrum of $R$. If $\operatorname{depth}(M)+\operatorname{depth}(N) \geq \operatorname{dim}(R)$ and $\operatorname{Tor}_{i}^{R}(M, N)=0$ for all $i>>$, then $M$ and $N$ satisfy the depth formula (1.1).

It seems reasonable to conjecture that if, in (1.4), we have $\operatorname{depth}(M)+\operatorname{depth}(N)<\operatorname{dim}(R)$, then $M \otimes_{R} N$ must have depth 0 . This is true if $R$ is the quotient of an unramified (or equicharacteristic) regular local ring by a nonzero element and $N$ has finite projective dimension. (See Lemma 2.3.)

1.5. Remark. Recently, Petra Constapel [C] has found a one-dimensional Gorenstein domain with two modules of depth 0 whose tensor product has depth 1 . The ring is $k\left[\left[t^{8}, \ldots, t^{14}\right]\right]$, which is not a complete intersection.

Hypersurfaces. A hypersurface is a ring of the form $S /(f)$, where $S$ is a regular local ring and $f$ is an element in the maximal ideal of $S$. For hypersurfaces, we are able to prove the depth formula under very mild hypotheses. There is one technicality that we will have to deal with. Suppose, for example, that $R=k[[x, y]]=k[[X, Y]] /(X Y)$, where $k$ is a field. Put $M=R /\left(x^{2}\right)$ and $N=R /(x)$. Then $x$ is a nonzero torsion element of $M$, since it is killed by the non-zero-divisor $x+y$. On the other hand $M \otimes_{R} N \cong R /(x) \cong(y)$, which is torsion-free (in fact, reflexive, as $R$ is onedimensional and Gorenstein). Thus $M$ has depth 0 , while $M \otimes_{R} N, N$ and $R$ all have depth 1 ; and the depth formula fails. We say that a finitely generated $R$-module ( $R$ any Noetherian ring) has constant rank (or constant rank $r$ ) provided there is an integer $r$ such that $M_{P} \cong R_{P}^{(r)}$ for every $P \in \operatorname{Ass}(R)$.

We recall the following results from [HW]:

1.6. THEOREM [HW, (2.7), (3.1)]. Let $R$ be a hypersurface, and let $M$ and $N$ be nonzero finitely generated $R$-modules, at least one of which has constant rank.

(1) If $M \otimes_{R} N$ is reflexive, then both $M$ and $N$ are reflexive, and $\operatorname{Tor}_{i}^{R}(M, N)=0$ for every $i>0$. 
(2) If $M \otimes_{R} N$ is a maximal Cohen-Macaulay module, then either $M$ or $N$ is free.

1.7. Corollary. Let $R$ be a hypersurface, and let $M$ and $N$ be nonzero finitely generated $R$-modules, at least one of which has constant rank. If $M \otimes_{R} N$ is reflexive, then $M$ and $N$ satisfy the depth formula (1.1).

Proof. By (1.6)(1) we have $\operatorname{Tor}_{i}^{R}(M, N)=0$ for all $i \geq 1$. The depth formula now follows from [HW, (2.5)].

The following example shows that in Corollary 1.7 it is not enough to assume that the tensor product is torsion-free.

1.8. ExAmple. Let $k$ be a field and put $R=k[[x, y, u, v]]=$ $k[[X, Y, U, V]] /(X Y-U V)$. Then $I:=(x, u), J:=(y, v)$ and $L:=(y, u)$ (and of course $R)$ are vector bundles of depth 3 . However, $\operatorname{depth}\left(I \otimes_{R} J\right)=0$, and $I \otimes_{R} L \cong \mathbf{m}:=(x, y, u, v)$, which has depth 1 . In particular, the depth formula fails in both cases.

Proof. This example is discussed in [HW, (4.1)], and it is shown there that $I, J$ and $L$ have depth 3 and that $I \otimes_{R} L$ is torsion-free. Therefore $I \otimes_{R} L \cong I L=\left(x y, x u, y u, u^{2}\right)=\left(u v, x u, y u, u^{2}\right)=u(v, x, y, u) \cong \mathbf{m}$. If $P$ is any non-maximal prime then $I_{P} \otimes_{R_{P}} L_{P} \cong \mathbf{m}_{P}=R_{P}$. It follows that $I$ and $L$ are vector bundles (and so is $J$ by symmetry). To see that $\operatorname{depth}\left(I \otimes_{R} J\right)=0$, that is, $I \otimes_{R} J$ is not torsion-free, we note [HW, (4.1)] that $\operatorname{Tor}_{2}^{R}(R / I, R / J) \neq 0$ and apply [HW, (1.4)].

By (1.4) we know that not all the higher Tors can vanish in the example above. In fact, it is shown in [HW, (4.1)] that $\operatorname{Tor}_{i}^{R}(I, J)=0$ if and only if $i$ is odd (or negative). Also $\operatorname{Tor}_{i}^{R}(I, L) \neq 0$ for every odd positive integer $i$. Surprisingly, vanishing of the higher Tors forces one of the modules to have finite projective dimension. This time there is a device for avoiding the hypothesis of constant rank.

1.9. Theorem. Let $R$ be a hypersurface, and let $M$ and $N$ be finitely generated $R$-modules. If $\operatorname{Tor}_{i}^{R}(M, N)=\operatorname{Tor}_{i+1}^{R}(M, N)=0$ for some $i \geq 0$, then either $M$ or $N$ has finite projective dimension.

Proof. We assume $M$ and $N$ are both nonzero. We write $R=S /(f)$, where $S$ is a regular local ring and use the long exact sequence (1.2.1) connecting $\mathrm{T}_{\bullet}^{S}:=$ Tor. $_{\bullet}^{S}$ and $\mathrm{T}_{\bullet}^{R}:=\operatorname{Tor}_{\bullet}^{R}$. We see that $\operatorname{Tor}_{i+1}^{S}(M, N)=0$, and the rigidity theorem of Auslander and Lichtenbaum [A], [L, Corollary 1] now implies that $\operatorname{Tor}_{j}^{S}(M, N)=0$ for all $j \geq i+1$. Now (1.2.1) shows that $\operatorname{Tor}_{j}^{R} \cong \operatorname{Tor}_{j+2}^{R}$ for all $j \geq i$, and it follows that $\operatorname{Tor}_{j}^{R}(M, N)=0$ for all $j \geq i$. (This is a special case of Murthy's rigidity theorem [M] for complete inter- 
sections, namely, the vanishing of $1+\operatorname{codim}(R)$ consecutive Tors forces all higher Tors to vanish; the assumption that the "ambient" regular local ring be unramified is no longer necessary in view of Lichtenbaum's proof [L] of rigidity in the ramified case.) By replacing $M$ and $N$ by sufficently high syzygies, we may assume that both $M$ and $N$ are maximal Cohen-Macaulay modules and that $\operatorname{Tor}_{i}^{R}(M, N)=0$ for all $i \geq 1$.

Our goal is to show that either $M$ or $N$ is free. We can harmlessly assume that neither $M$ nor $N$ has a nonzero free summand, and now we seek a contradiction. Since (for complete intersections) the depth formula holds whenever all positive Tors vanish [HW, (2.5)], $M \otimes_{R} N$ is a maximal CohenMacaulay module. Since we are not assuming that $M$ or $N$ has constant rank, we need to pass to the double branched cover $R^{\#}:=S[[Z]] /\left(f+Z^{2}\right)$, [HW, $-\mathrm{p} 3]$. On reading through the proof of [HW, (3.6)] we discover that the hypothesis that $M$ have constant rank is never used. Thus (3.6) implies that

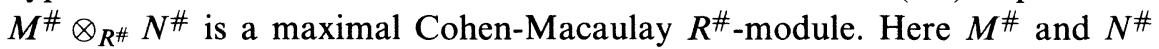

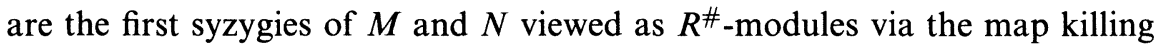
$z$. Being syzygies of torsion modules, $M^{\#}$ and $N^{\#}$ have constant rank. By (1.6)(2) either $M^{\#}$ or $N^{\#}$ is free. But [HW, (3.5)] says that neither $M^{\#}$ nor $N^{\#}$ has a nonzero free summand. Thus either $M^{\#}$ or $N^{\#}$ is 0 , an obvious contradiction.

A particular example of this theorem was used by Dutta, Hochster, and McLaughlin in [DHM]. Claudia Miller, a student at the University of Illinois, has given a nice proof of this theorem without appealing to Theorem 1.6.

\section{Local cohomology.}

Let $(R, \mathbf{m}, k)$ be a local ring. Given a finitely generated $R$-module $M$, let $\mathrm{H}_{\mathrm{m}}^{0}(M)=\left\{x \in M \mid \mathbf{m}^{t} x=0\right.$ for some $\left.t \geq 1\right\}$. Then $\mathrm{H}_{\mathrm{m}}^{0}$ is a left exact functor on $R$-modules, and by definition $\left\{\mathrm{H}_{\mathrm{m}}^{i}\right\}$ is its sequence of right derived functors. If $M$ is finitely generated and nonzero, then [BH, (3.5.7)] depth $(M)$ (respectively $\operatorname{dim}(M)$ ) is the least (respectively largest) integer $r$ such that $\mathrm{H}_{\mathrm{m}}^{r}(M) \neq 0$. Thus the vanishing of $\mathrm{H}_{\mathrm{m}}^{i}(M)$ for $i \leq r$ is equivalent to the depth of $M$ being at least $r+1$. Our main results in this section are essentially rigidity theorems for the local cohomology of a tensor product of modules. For example, (2.4) says (for hypersurfaces with at least one of the modules having finite projective dimension) that the vanishing of $\mathrm{H}_{\mathrm{m}}^{r}\left(M \otimes_{R} N\right)$ entails the vanishing of all lower local cohomology modules. Thus, the vanishing of a single local cohomology module gives a lower bound on the depth. A similar result (2.5), for hypersurfaces without assuming one of the modules has finite projective dimension, assumes that two 
consecutive local cohomology modules vanish. We were motivated to begin study of this problem by a result of Luk and Yau [LY] which gave some conditions which imply holomorphic vector bundles over complex projective space are direct sums of line bundles. Suitably interpreted, their conditions amount to the condition that $\mathrm{H}_{\mathrm{m}}^{2}\left(E \otimes E^{*}\right)=0$, for a module $E$ corresponding to the vector bundle, and their conclusion corresponds to the statement that $E$ is free. We derive this from our main theorem, which is considerably more general. We discuss applications to vector bundles in Section 5 .

2.1. Lemma. Let $(R, \mathbf{m})$ be a local Gorenstein ring and let $M$ be a finitely generated $R$-module. Let $r$ be an integer, $1 \leq r<\operatorname{dim} R$. Assume that depth $M \geq r$ and $M$ satisfies Serre's condition $\left(\mathrm{S}_{\mathrm{n}}\right)$ on the punctured spectrum of $R$, for some $n \geq 1$. Then there is an exact sequence

$$
0 \rightarrow M \rightarrow F \rightarrow C \rightarrow 0,
$$

in which

(1) $F$ is free,

(2) $C$ has depth at least $r-1$,

(3) C satisfies $\left(\mathrm{S}_{\mathrm{n}-1}\right)$ on the punctured spectrum, and

(4) for every prime ideal $p, C_{p}$ is free iff $M_{p}$ is free.

Proof. Let $F$ be a free $R$-module of rank at least $\mu_{R}\left(M^{*}\right):=$ number of generators required for $M^{*}$. Map $F^{*}$ onto $M^{*}$, obtaining a short exact sequence

$$
0 \rightarrow X \rightarrow F^{*} \rightarrow M^{*} \rightarrow 0 .
$$

Dualize, getting an exact sequence

$$
0 \rightarrow M^{* *} \rightarrow F \rightarrow X^{*} \rightarrow \operatorname{Ext}_{R}^{1}\left(M^{*}, R\right) \rightarrow 0 .
$$

$M$ is torsion-free since both $r$ and $n$ are at least 1 , so that $M$ embeds in $M^{* *}$. This gives an exact sequence,

$$
0 \rightarrow M \rightarrow F \rightarrow C \rightarrow 0 .
$$

The exact sequence (2.1.3) is called the universal push forward of $M$. It has two properties: firstly it commutes with localization (up to free summands), and secondly if $M$ is an $n$th syzygy, then $C$ is an $(n-1)^{\text {th }}$ syzygy. The localization property is clear from the construction, while the syzygy property is explained on page 49 of [EG]. Since $R$ is Gorenstein, it then follows from Theorem 3.8 of [EG] that as $M$ is $\left(\mathrm{S}_{\mathrm{n}}\right)$ on the punctured spectrum, $C$ must be $\left(\mathrm{S}_{\mathrm{n}-1}\right)$ on the punctured spectrum. The exact sequence (2.1.3) shows that the depth of $C$ must be at least depth $M-1$, which gives (2); and (4) is clear from the construction. 
2.2. Lemma. Let $M$ and $N$ be finitely generated modules over a Noetherian local ring $(R, \mathbf{m})$ such that the projective dimension of $N$ is finite. Let $q$ be the largest integer such that $\operatorname{Tor}_{q}^{R}(M, N) \neq 0$. If the depth of $\operatorname{Tor}_{q}^{R}(M, N)$ is at most one, or if $q=0$, then

$$
\text { depth } M+\operatorname{depth} N=\operatorname{depth} R+\operatorname{depth}\left(\operatorname{Tor}_{q}^{R}(M, N)\right)-q .
$$

Proof. This is simply the statement of $[A 1,(1.2)]$ after taking into account the Auslander-Buchsbaum formula.

2.3. Lemma. Let $(R, \mathbf{m})$ be a local ring whose completion is the quotient of an unramified (or equicharacteristic) regular local ring $S$ by a nonzero element. Let $M$ and $N$ be finitely generated R-modules such that $M \otimes_{R} N$ is torsion-free. Assume in addition that $\operatorname{pd}_{R}(N)<\infty$. Then $\operatorname{Tor}_{i}^{R}(M, N)=0$ for all $i \geq 1$ and the depth formula holds for $M$ and $N$.

Proof. ${ }^{1}$ We may assume that $R$ is complete. ${ }^{2}$ Let $\bar{M}$ (respectively $\bar{N}$ ) be $M$ modulo its torsion (respectively $N$ modulo torsion). An easy argument (See Lemma 1.1 of [HW]) shows that $\bar{M} \otimes_{R} N$ is also torsion-free. Embed $\bar{M}$ into a free $R$-module $F$, and let $C$ be the cokernel. Tensoring with $N$ gives an exact sequence,

$$
0 \rightarrow \operatorname{Tor}_{1}^{R}(C, N) \rightarrow \bar{M} \otimes_{R} N \rightarrow \ldots
$$

Since $\bar{M} \otimes_{R} N$ is torsion-free, it follows that $\operatorname{Tor}_{1}^{R}(C, N)=0$ as this module is torsion ( $N$ has finite projective dimension and is therefore generically free). Applying Theorem 3 of [L], we obtain that $\operatorname{Tor}_{i}^{R}(C, N)=0$ for all $i \geq 1$. It follows that $\operatorname{Tor}_{i}^{R}(\bar{M}, N)=0$ for all $i \geq 1$. Let $\tau \subseteq M$ be the torsion submodule of $M$. Tensoring the exact sequence $0 \rightarrow \tau \rightarrow M \rightarrow \bar{M} \rightarrow 0$ with $N$, we then see that $\tau \otimes_{R} N \subseteq M \otimes_{R} N$. The second module is torsion-free, forcing $\tau=0$. The vanishing statement of the Lemma follows immediately, while the last statement follows from [HW, (2.5)].

We are ready to prove one of our main results.

2.4. ThEOREM. Let $M$ and $N$ be finitely generated nonzero modules over a

${ }^{1}$ This Lemma is essentially due to Lichtenbaum. One only needs to put together some of Auslander's ideas with Theorem 3 in [L]. Namely, in Theorem 3 of [L], Lichtenbaum proves rigidity of Tor for any two finitely generated modules over $R$ such that all sufficiently high Tors vanish. As we have seen in Theorem 1.9, the assumption of finite projective dimension for one of the two modules, a clearly sufficient condition for the higher Tors to vanish, is also necessary.

${ }^{2}$ There is a technical point in the reduction to the complete case: We need to know that $M \otimes_{R} N \otimes_{R} \hat{R}$ is torsion-free. Note that the hypotheses on $R$ imply that $R$ is Gorenstein and therefore that $M \otimes_{R} N$ can be embedded into a free $R$-module. On completing, we see that $M \otimes_{R} N \otimes_{R} \hat{R}$ embeds into a free $\hat{R}$-module and is therefore torsion-free. 
local ring $(R, \mathbf{m})$ of dimension $d$ whose completion is the quotient of an unramified (or equicharacteristic) regular local ring by a nonzero element. Let $r$ be an integer with $0 \leq r<d$. Assume that $M \otimes_{R} N$ satisfies Serre's condition $\left(\mathrm{S}_{\mathrm{r}+1}\right)$ on the punctured spectrum of $R$. Further assume that $N$ has finite projective dimension. The following conditions are equivalent:

(1) $\mathrm{H}_{\mathbf{m}}^{r}\left(M \otimes_{R} N\right)=0$ and both $M$ and $N$ have depth at least $r$.

(2) $\operatorname{depth}(M)+\operatorname{depth}(N) \geq d+r+1$.

(3) $\operatorname{depth}\left(M \otimes_{R} N\right) \geq r+1$.

(4) $M \otimes_{R} N$ satisfies Serre's condition $\left(\mathrm{S}_{\mathrm{r}+1}\right)$.

Proof. Since $M \otimes_{R} N$ satisfies ( $\left.\mathrm{S}_{\mathrm{r}+1}\right)$ on the punctured spectrum, (3) and (4) are clearly equivalent. Assume (4). $M \otimes_{R} N$ satisfies $\left(\mathrm{S}_{1}\right)$, that is, thetensor product is torsion-free. Lemma 2.3 then proves the depth formula holds for $M$ and $N$, which implies condition (2).

Assume (2). Since $M \otimes_{R} N$ is torsion-free on the punctured spectrum, Lemma 2.3 applies to prove that all the higher Tors of $M$ and $N$ are zero on the punctured spectrum, i.e., that $\operatorname{Tor}_{i}^{R}(M, N)$ has finite length for $i \geq 1$. If some $\operatorname{Tor}_{i}^{R}(M, N)$ is nonzero for some $i \geq 1$, then we may apply Lemma 2.2 to reach the contradiction that $-q \geq r+1$, where $q$ is the index of the last nonvanishing Tor. Hence $\operatorname{Tor}_{i}^{R}(M, N)=0$ for $i \geq 1$. By [HW, (2.5)] the depth formula holds and $\operatorname{depth}\left(M \otimes_{R} N\right)=$ depth $M+$ depth $N-d \geq r+1$. Hence $\mathrm{H}_{\mathrm{m}}^{r}\left(M \otimes_{R} N\right)=0$. Both $M$ and $N$ must also have depth at least $r$, as each of their depths is bounded by $d$. This proves (1).

Now assume (1) holds. If $r=0$ we get (3) immediately. Assume $r>0$ and proceed by induction. Since $N$ has constant rank (1.7) implies that the depth formula holds on the punctured spectrum for $M$ and $N$. In particular, both $M$ and $N$ must be $\left(\mathrm{S}_{\mathrm{r}+1}\right)$ on the punctured spectrum (see [HW, Corollary 2.6]). Therefore they satisfy $\left(S_{\mathrm{r}}\right)$ since (1) gives that their depths are at least $r$. Form the universal pushforward as in Lemma 2.1:

$$
0 \rightarrow M \rightarrow F \rightarrow C \rightarrow 0,
$$

in which $F$ is free, $C$ has depth at least $r-1$, and $C$ satisfies $\left(\mathrm{S}_{\mathrm{r}}\right)$ on thepunctured spectrum.

Tensoring (2.4.1) with $N$, we get a four-term exact sequence

$$
0 \rightarrow \operatorname{Tor}_{1}^{R}(C, N) \rightarrow M \otimes_{R} N \rightarrow F \otimes_{R} N \rightarrow C \otimes_{R} N \rightarrow 0,
$$

which we break into two short exact sequences:

$$
\begin{aligned}
& 0 \rightarrow \operatorname{Tor}_{1}^{R}(C, N) \rightarrow M \otimes_{R} N \rightarrow D \rightarrow 0, \\
& 0 \rightarrow D \rightarrow F \otimes_{R} N \rightarrow C \otimes_{R} N \rightarrow 0 .
\end{aligned}
$$


We claim that $T:=\operatorname{Tor}_{1}^{R}(C, N)$ has finite length. The module $N$ is generically free since it has finite projective dimension. In particular, $\operatorname{Tor}_{1}^{R}(C, N)$ is torsion. As $M \otimes_{R} N$ is torsion-free on the punctured spectrum of $R$ the claim follows. In particular $\mathrm{H}_{\mathrm{m}}^{r+1}(T)=0$. It follows from the long exact sequence of local cohomology (applied to (2.4.3)) that $\mathrm{H}_{\mathbf{m}}^{r}(D)=0$. Also, since $\operatorname{depth}(N) \geq r$, we have $\mathrm{H}_{\mathrm{m}}^{r-1}(N)=0$. Part of the long exact sequence of local cohomology from (2.4.4) is:

$$
\ldots \rightarrow \mathrm{H}_{\mathbf{m}}^{r-1}\left(F \otimes_{R} N\right) \rightarrow \mathrm{H}_{\mathbf{m}}^{r-1}\left(C \otimes_{R} N\right) \rightarrow \mathrm{H}_{\mathbf{m}}^{r}(D) \rightarrow \ldots,
$$

which proves that $\mathrm{H}_{\mathrm{m}}^{r-1}\left(C \otimes_{R} N\right)=0$. We claim that $C \otimes_{R} N$ satisfies $\left(\mathrm{S}_{\mathrm{r}}\right)$ on the punctured spectrum. Localizing at a prime $p \neq \mathbf{m}$ we get an exact sequence,

$$
0 \rightarrow\left(M \otimes_{R} N\right)_{p} \rightarrow\left(F \otimes_{R} N\right)_{p} \rightarrow\left(C \otimes_{R} N\right)_{p} \rightarrow 0 .
$$

Both $\left(M \otimes_{R} N\right)_{p}$ and $N_{p}$ satisfy $\left(\mathrm{S}_{\mathrm{r}+1}\right)$ (see the discussion before (2.4.1)). If $\operatorname{dim} R_{p} \geq r+1$, the exact sequence above shows that $\left(C \otimes_{R} N\right)_{p}$ has depth at least $r$. If $\operatorname{dim} R \leq r,\left(M \otimes_{R} N\right)_{p}$ will be maximal Cohen-Macaulay. In this case both $M_{p}$ and $N_{p}$ will be maximal Cohen-Macaulay by Lemma 2.3 and [HW, (2.0)]. Since $N_{p}$ has finite projective dimension, $N_{p}$ will be free. Since $C_{p}$ satisfies $\left(\mathrm{S}_{\mathrm{r}}\right),\left(C \otimes_{R} N\right)_{p}$ is also maximal Cohen-Macaulay, which finishes the proof that $C \otimes_{R} N$ satisfies $\left(\mathrm{S}_{\mathrm{r}}\right)$ on the punctured spectrum.

Our inductive assumption then gives that $\operatorname{depth}\left(C \otimes_{R} N\right) \geq r$. In particular, $C \otimes_{R} N$ is torsion-free (since $r>0$ ), and we have $\operatorname{Tor}_{1}^{R}(C, N)=0$ by Lemma 2.3. Now (2.4.2) proves that $\operatorname{depth}\left(M \otimes_{R} N\right) \geq r+1$.

Note that every complete ramified regular local ring is the quotient of an unramified regular local ring by a nonzero element. Thus Theorem 2.4 applies to all regular local rings.

The modules in (1.8) show that (2) does not imply (3) if $N$ is not assumed to have finite projective dimension. Also, when viewed as modules over the regular local ring $k[[X, Y, U, V]]$, they show that the Serre condition on the punctured spectrum cannot be eliminated.

Next we prove another rigidity theorem for hypersurfaces, in which neither of the modules is assumed to be finite projective dimension. Not surprisingly, in this case we require that two consecutive local cohomology modules vanish. After the fact, this then forces one of the modules to be of finite projective dimension, and the equivalences below can in part be viewed as giving sufficient conditions for a module to have finite projective dimension.

2.5. Theorem. Let $(R, \mathbf{m})$ be a hypersurface of dimension $d$. Let $M$ and $N$ be finitely generated nonzero modules over $R$, at least one of which has con- 
stant rank. Let $r$ be an integer with $1 \leq r<d$. Assume that $M \otimes_{R} N$ satisfies Serre's condition $\left(\mathrm{S}_{\mathrm{r}+1}\right)$ on the punctured spectrum of $R$. The following conditions are equivalent:

(1) $\mathrm{H}_{\mathrm{m}}^{r-1}\left(M \otimes_{R} N\right)=\mathrm{H}_{\mathrm{m}}^{r}\left(M \otimes_{R} N\right)=0$ and both $M$ and $N$ have depth at least $r$.

(2) $\operatorname{depth}(M)+\operatorname{depth}(N) \geq d+r+1$, and either $M$ or $N$ has finite projective dimension.

(3) $\operatorname{depth}\left(M \otimes_{R} N\right) \geq r+1$.

(4) $M \otimes_{R} N$ satisfies Serre's condition $\left(\mathrm{S}_{\mathrm{r}+1}\right)$.

Proof. This proof is almost identical to that of Theorem 2.4. However, it is difficult to combine the two proofs efficiently. We may assume that $M$ has constant rank. Since $M \otimes_{R} N$ satisfies $\left(\mathrm{S}_{\mathrm{r}+1}\right)$ on the punctured spectrum, (3) and (4) are clearly equivalent. Assume (3). Then $M \otimes_{R} N$ satisfies $\left(\mathrm{S}_{2}\right)$, that is, the tensor product is reflexive. By Theorems 1.6 and 1.9 , either $M$ or $N$ has finite projective dimension. Corollary 1.7 proves the depth formula holds for $M$ and $N$, which implies condition (2).

Assume (2). Since $M \otimes_{R} N$ is reflexive on the punctured spectrum, Theorem 1.6 applies to prove that all the higher Tors of $M$ and $N$ are zero on the punctured spectrum, i.e., that $\operatorname{Tor}_{i}^{R}(M, N)$ has finite length for $i \geq 1$. If some $\operatorname{Tor}_{i}^{R}(M, N)$ is nonzero for some $i \geq 1$, then we may apply Lemma 2.2 to reach the contradiction that $-q \geq r+1$, where $q$ is the index of the last nonvanishing Tor. Hence $\operatorname{Tor}_{i}^{R}(M, N)=0$ for $i \geq 1$. Then the depth formula holds [HW, (2.5)] and $\operatorname{depth}\left(M \otimes_{R} N\right)=\operatorname{depth} M+\operatorname{depth} N-d \geq r+1$. Hence $\mathrm{H}_{\mathrm{m}}^{r}\left(M \otimes_{R} N\right)=0$. Both $M$ and $N$ must also have depth at least $r$, as each of their depths is bounded by $d$. This proves (1).

Now assume (1) holds. If $r=1$ we get (3) immediately. Assume $r>1$ and proceed by induction. As $M \otimes_{R} N$ is reflexive on the punctured spectrum, Corollary 1.7 shows that the depth formula holds on the punctured spectrum for $M$ and $N$. In particular, both $M$ and $N$ must be $\left(\mathrm{S}_{\mathrm{r}+1}\right)$ on the punctured spectrum (see [HW, Corollary 2.6]). Hence both $M$ and $N$ satisfy $\left(\mathrm{S}_{\mathrm{r}}\right)$ since (1) gives that their depths are at least $r$. There is a short exact sequence given by Lemma 2.1 :

$$
0 \rightarrow M \rightarrow F \rightarrow C \rightarrow 0,
$$

in which $F$ is free, $C$ has depth at least $r-1$, and $C$ satisfies $\left(\mathrm{S}_{\mathrm{r}}\right)$ on the punctured spectrum. Furthermore, $C$ will also have constant rank.

Tensoring (2.5.1) with $N$, we get a four-term exact sequence

$$
0 \rightarrow \operatorname{Tor}_{1}^{R}(C, N) \rightarrow M \otimes_{R} N \rightarrow F \otimes_{R} N \rightarrow C \otimes_{R} N \rightarrow 0
$$

which we break into two short exact sequences: 


$$
\begin{aligned}
& 0 \rightarrow \operatorname{Tor}_{1}^{R}(C, N) \rightarrow M \otimes_{R} N \rightarrow D \rightarrow 0, \\
& 0 \rightarrow D \rightarrow F \otimes_{R} N \rightarrow C \otimes_{R} N \rightarrow 0 .
\end{aligned}
$$

We claim that $T:=\operatorname{Tor}_{1}^{R}(C, N)$ has finite length. Since $C$ has constant rank we know that $\operatorname{Tor}_{1}^{R}(C, N)$ is torsion. As $M \otimes_{R} N$ is torsion-free on the punctured spectrum of $R$ the claim follows. In particular $H_{\mathrm{m}}^{i}(T)=0$ for $i \geq 1$. It follows from the long exact sequence of local cohomology (applied to (2.5.3)) that $\mathrm{H}_{\mathbf{m}}^{i}(D)=0$ for $i=r-1, r$. Also, since depth $(N) \geq r$, we have $\mathrm{H}_{\mathrm{m}}^{j}(N)=0$ for $j \leq r-1$. Part of the long exact sequence of local cohomology from (2.5.4) is:

$$
\ldots \rightarrow \mathrm{H}_{\mathbf{m}}^{j}(N) \rightarrow \mathrm{H}_{\mathbf{m}}^{j}\left(C \otimes_{R} N\right) \rightarrow \mathrm{H}_{\mathbf{m}}^{j+1}(D) \rightarrow \ldots,
$$

which proves that $\mathrm{H}_{\mathrm{m}}^{j}\left(C \otimes_{R} N\right)=0$ for $j=r-2, r-1$. Lemma 2.1 shows that depth $(C) \geq r-1$. We claim that $C \otimes_{R} N$ satisfies $\left(\mathrm{S}_{\mathrm{r}}\right)$ on the punctured spectrum. Localizing at a prime $p \neq \mathbf{m}$, we get an exact sequence

$$
0 \rightarrow\left(M \otimes_{R} N\right)_{p} \rightarrow\left(F \otimes_{R} N\right)_{p} \rightarrow\left(C \otimes_{R} N\right)_{p} \rightarrow 0 .
$$

If $\operatorname{dim} R_{p} \geq r+1$, then $\operatorname{depth}\left(M \otimes_{R} N\right)_{p} \geq r+1$, and by Corollary 1.7 it follows that both $M_{p}$ and $N_{p}$ have depth at least $r+1$. The exact sequence above then proves that the depth of $\left(C \otimes_{R} N\right)_{p}$ is at least $r$. If $\operatorname{dim} R_{p} \leq r$, $\left(M \otimes_{R} N\right)_{p}$ will be maximal Cohen-Macaulay. In this case [HW, (3.1) says that either $M_{p}$ or $N_{p}$ is free. If $M$ is free so is $C_{p}$ by Lemma 2.1. If $N_{p}$ is free then $\operatorname{depth}\left(C \otimes_{R} N\right)_{p}=\operatorname{depth} C_{p}=\operatorname{dim} R_{p}$ (since $C$ satisfies $\left(\mathrm{S}_{\mathrm{r}}\right)$ on the punctured spectrum). This finishes the proof that $C \otimes_{R} N$ satisfies $\left(\mathrm{S}_{\mathrm{r}}\right)$ on the punctured spectrum.

Our inductive assumption then gives that $\operatorname{depth}\left(C \otimes_{R} N\right) \geq r$. In particular, $C \otimes_{R} N$ is reflexive (since $r>1$ ), and we have $\operatorname{Tor}_{1}^{R}(C, N)=0$ by Theorem 1.6. Now (2.5.2) proves that $\operatorname{depth}\left(M \otimes_{R} N\right) \geq r+1$.

In the next section we will give several applications of these theorems to questions concerning the depth of tensor products of vector bundles, and when vector bundles are free. To close this section, we give an application of Theorem 2.4 to give a quick proof of the main technical result of the paper of Auslander and Goldman [AG, Theorem 4.4]:

2.6. CoRollary. Let $(R, \mathbf{m})$ be a regular local ring and let $M$ be a finitely generated $R$-module such that $\operatorname{Hom}_{R}(M, M)$ is a free $R$-module. Then $M^{* *}$ is free.

Proof. Since $R$ is integrally closed, a standard argument shows that the double dual of $\operatorname{Hom}_{R}(M, M)$ is $\operatorname{Hom}_{R}\left(M^{* *}, M^{* *}\right)$ (cf. [AG, (4.1)]). In particular, we may assume that $M$ is reflexive. By induction on the dimension of 
$R$ we may also assume that $M$ is free on the punctured spectrum and that $\operatorname{dim} R \geq 3$ (since reflexive modules over a 2-dimensional regular local ring are free by the Auslander-Buchsbaum formula). It follows that $\mathrm{H}_{\mathrm{m}}^{2}(R)=0$ and therefore $\mathrm{H}_{\mathrm{m}}^{2}\left(\operatorname{Hom}_{R}(M, M)\right)=0$.

There is a canonical map from $M \otimes_{R} M^{*} \rightarrow \operatorname{Hom}_{R}(M, M)$ which is an isomorphism iff $M$ is free (cf. [A1, (3.3)]). In our situation, both the kernel and cokernel of this canonical map will be of finite length, and consequently $\mathrm{H}_{\mathrm{m}}^{2}\left(M \otimes_{R} M^{*}\right)=0$. Theorem 2.4 applies to give that $M \otimes_{R} M^{*}$ is reflexive, and now an elementary argument given in [A, (3.3)] proves that $M$ is free. $^{3}$

Our main theorem can also be used to prove that modules of the form $\operatorname{Hom}_{R}(M, N)$ rarely have good depth properties. Namely:

2.7. Corollary. Let $(R, \mathbf{m})$ be a local ring of dimension $d$ whose completion is the quotient of an unramified (or equicharacteristic) regular local ring by a nonzero element. Let $M$ and $N$ be finitely generated reflexive $R$-modules, such that $N$ has finite projective dimension. Suppose that $\operatorname{Hom}_{R}(M, N)$ satisfies Serre's condition $\left(\mathrm{S}_{3}\right)$. Then $\operatorname{Tor}_{i}^{R}\left(M^{*}, N\right)=0$ for all $i \geq 1, M^{*}$ and $N$ satisfy the depth formula, and $M^{*} \otimes_{R} N \cong \operatorname{Hom}_{R}(M, N)$ via the natural map.

Proof. We induce upon the dimension of $R$. If $\operatorname{dim}(R) \leq 2$, then $N$ is free, and in that case the Corollary is immediate. There is a natural map $\phi: M^{*} \otimes_{R} N \rightarrow \operatorname{Hom}_{R}(M, N)$ given by $f \otimes n \rightarrow\{m \rightarrow f(m) n\}$. By induction we may assume that the kernel and cokernel of $\phi$ have finite length. In this case $\mathrm{H}_{\mathrm{m}}^{2}\left(M^{*} \otimes_{R} N\right)=\mathrm{H}_{\mathrm{m}}^{2}\left(\operatorname{Hom}_{R}(M, N)\right)=0$. On the punctured spectrum, $M^{*} \otimes_{R} N$ satisfies $\left(\mathrm{S}_{3}\right)$ since it is isomorphic with $\operatorname{Hom}_{R}(M, N)$ which satisfies $\left(\mathrm{S}_{3}\right)$. Moreover the depths of $M$ and $N$ are at least 2 since they are reflexive and $\operatorname{dim}(R) \geq 3$. Theorem 2.4 then gives that $M^{*} \otimes_{R} N$ satisfies $\left(\mathrm{S}_{3}\right)$. It then follows from (1.6) that $\operatorname{Tor}_{i}^{R}\left(M^{*}, N\right)=0$ for all $i \geq 1$, and since both $M^{*} \otimes_{R} N$ and $\operatorname{Hom}_{R}(M, N)$ satisfy $\left(\mathrm{S}_{3}\right), \phi$ must be an isomorphism. Finally $M^{*}$ and $N$ satisfy the depth formula by (1.7).

2.8. Corollary. Let $(R, \mathrm{~m})$ be a local ring of dimension $d$ whose completion is the quotient of an unramified (or equicharacteristic) regular local ring by a nonzero element. Let $M$ and $N$ be finitely generated reflexive $R$-modules, such that $N$ has finite projective dimension and is nonzero. Suppose that $\operatorname{Hom}_{R}(M, N)$ satisfies Serre's condition $\left(\mathrm{S}_{3}\right)$ and that a finite direct sum of copies of $M$ maps onto $N$. Then $M$ has a nonzero free summand.

\footnotetext{
${ }^{3}$ The general statement is that if $R$ is an integrally closed domain, and $M$ is a torsion-free $R$ module such that $M \otimes_{R} M^{*}$ is reflexive, then $M$ is projective. One proves this by considering the natural map from $M \otimes_{R} M^{*} \rightarrow \operatorname{Hom}_{R}(M, M)$ which is an isomorphism iff $M$ is projective. By induction on the dimension one can assume the kernel and cokernel of this map have finite length. But then $M \otimes_{R} M^{*}$ reflexive gives that the map is an isomorphism.
} 
Proof. Our assumptions guarantee that $M^{*} \otimes_{R} N \cong \operatorname{Hom}_{R}(M, N)$ by (2.7), and that $\oplus M \rightarrow N$ is onto for some finite direct sum of copies of $M$. It then follows that the natural map $\phi: M \otimes_{R} \operatorname{Hom}_{R}(M, N) \rightarrow N$ induced by $m \otimes f \rightarrow f(m)$ is onto. Consequently the map from $M \otimes_{R} M^{*} \otimes_{R} N$ to $N$ is onto. However, this map has image in $\tau N$, where $\tau$ is the trace ideal, i.e. the image of the natural map from $M \otimes_{R} M^{*}$ to $R$. If $\tau \subset \mathbf{m}$ then $N \subset \mathbf{m} N$, which contradicts Nakayama's lemma. Thus $\tau=R$, and since $R$ is local it follows that $M$ maps onto $R$.

2.9. Corollary. Let $(R, \mathbf{m})$ be a regular local ring, and let $M$ be a finitely generated reflexive $R$-module. If $\operatorname{Hom}_{R}(M, M)$ satisfies Serre's condition $\left(\mathrm{S}_{3}\right)$, then $M$ is free.

Proof. Induce upon the dimension of $R$. If $\operatorname{dim}(R) \leq 2$ then $M$ is free since it is reflexive. We may assume that $M$ is free on the punctured spectrum of $R$, and that $\operatorname{dim}(R) \geq 3$. Corollary (2.7) gives that $M^{*}$ and $M$ satisfy the depth formula:

$$
\text { depth } M^{*}+\operatorname{depth} M \geq \operatorname{dim} R+3,
$$

We can now apply duality $[\mathrm{V},(3.3 .16)]$ to conclude that $M$ is free.

\section{Tensor Products of Vector Bundles over a Regular Local Ring.}

3.0. Remark. Let $(R, \mathbf{m})$ be a local ring. By a slight abuse of terminology, we define a vector bundle over $R$ to be a finitely generated $R$-module $M$ such that $M_{P}$ is a free $R_{P}$-module for every prime ideal $P \neq \mathbf{m}$. Note that we do not require $M$ to be reflexive; thus there may be many non-isomorphic vector bundles over $R$ that induce the same vector bundle (= coherent locally free sheaf) on the punctured spectrum $\operatorname{Spec}(R)-\{\mathbf{m}\}$.

Our starting point in this section is the observation that the equivalence of (2) and (3) in (2.4) gives a formula for the depth of the tensor product of two vector bundles in terms of the depths of the factors. An easy induction yields the following generalization of this formula:

3.1. Proposition. Let $M_{1}, M_{n}$ be nonzero vector bundles over a regular local ring $R$ of dimension $d$. Then $\operatorname{depth}\left(M_{1} \otimes_{R} \ldots \otimes_{R} M_{n}\right)=$ $\max \left\{0, \sum_{i=1}^{n} \operatorname{depth}\left(M_{i}\right)-(n-1) d\right\}$.

Every regular local ring has a nice source of vector bundles of various depths, namely the syzygies of the residue field. Given a regular local ring $(R, \mathbf{m}, k)$, let $E_{r}$ denote the $r$ th syzygy in the minimal (Koszul) resolution of $k$. Then $\operatorname{depth}\left(E_{r}\right)=r$ for $0 \leq i \leq d$. In particular we have the tangent bundle $T:=E_{d-1}$. Using (3.1) it is easy to compute the depth of any tensor power of $T:$ 
3.2. EXAMPLE. Let $R$ be a regular local ring of dimension $d$, and let $T$ be the tangent bundle. Then $\operatorname{depth}\left(\otimes_{R}^{n} T\right)=\max \{0, d-n\}$.

In order to get information from the vanishing of local cohomology of an $n$-fold tensor product, we will use the following lemma, whose proof is an easy diagram chase left to the reader.

3.3. Lemma. Let $M$ be a vector bundle over a local ring $(R, \mathbf{m})$, and let $\bar{M}$ be the image of the natural map from $M$ into its double dual $M^{* *}$. Let $N$ be any finitely generated $R$-module.

(1) The natural map $\mathrm{H}_{\mathrm{m}}^{i}\left(M \otimes_{R} N\right) \rightarrow \mathrm{H}_{\mathrm{m}}^{i}\left(\bar{M} \otimes_{R} N\right)$ is surjective for $i=0$ and an isomorphism for $i \geq 1$.

(2) The natural map $\mathrm{H}_{\mathrm{m}}^{i}\left(M \otimes_{R} N\right) \rightarrow \mathrm{H}_{\mathrm{m}}^{i}\left(M^{* *} \otimes_{R} N\right)$ is surjective for $i=1$ and an isomorphism for $i \geq 2$.

3.4. Proposition. Let $M_{1}, \ldots, M_{n}$ be nonzero torsion-free vector bundles over a regular local ring $(R, \mathbf{m})$ of dimension $d \geq 2$, and suppose $\mathrm{H}_{\mathbf{m}}^{1}\left(M_{1} \otimes_{R} \ldots \otimes_{R} M_{n}\right)=0$.

(1) $M_{i_{1}} \otimes_{R} \ldots \otimes_{R} M_{i_{t}}$ is reflexive whenever $1 \leq i_{1}<\ldots<i_{t} \leq n$.

(2) $\sum_{i=1}^{n} \operatorname{depth}\left(M_{i}\right)=(n-1) d+\operatorname{depth}\left(M_{1} \otimes_{R} \ldots \otimes_{R} M_{n}\right) \geq(n-1) d+2$.

(3) If $n \geq d-1$ then at least one of the $M_{i}$ is free.

Proof. The case $n=1$ is trivial, and if $n=2$ we can apply (2.4). Therefore we assume $n \geq 3$ and proceed inductively. Put $N=M_{2} \otimes_{R} \ldots \otimes_{R} M_{n}$. By (3.3) $\mathrm{H}_{\mathrm{m}}^{1}\left(M_{1} \otimes_{R} \bar{N}\right)=0$, and (1.4) then implies that $\operatorname{depth}\left(M_{1}\right)+$ $\operatorname{depth}(\bar{N}) \geq d+2$. Therefore $\operatorname{depth}(\bar{N}) \geq 2$. Then $\mathrm{H}_{\mathbf{m}}^{1}(\bar{N})=0$, so $\mathrm{H}_{\mathbf{m}}^{1}(N)=0$ by (3.3). By induction (and permutation of the subscripts), we see that

$$
\mathrm{H}_{\mathbf{m}}^{1}\left(M_{i_{1}} \otimes_{R} \ldots \otimes_{R} M_{i_{t}}\right)=0 \text { if } 1 \leq i_{1}<\ldots<i_{t} \leq n .
$$

Applying (1) to $N$ with $t=n-1$, we see that $N$ is reflexive and therefore torsion-free. Then (2.4)(4) implies that $M \otimes_{R} N$ is reflexive, and now (1) follows from (3.4.1) and induction.

The inequality in (2) is now clear, and the identity follows from (3.1).

If none of the $M_{i}$ is free then $\sum_{i=1}^{n} \operatorname{depth}\left(M_{i}\right) \leq n(d-1)$. Then (2) implies that $n(d-1) \geq(n-1) d+2$, that is, $n \leq d-2$. This proves (3) and completes the proof.

Using essentially the same proof, with $N^{* *}$ replacing $\bar{N}$, we obtain the following:

3.5. Proposition. Let $M_{1}, \ldots, M_{n}$ be nonzero reflexive vector bundles over a regular local ring $(R, \mathbf{m})$ of dimension $d \geq 3$, and suppose $\mathrm{H}_{\mathbf{m}}^{2}\left(M_{1} \otimes_{R} \ldots \otimes_{R} M_{n}\right)=0$.

(1) $\operatorname{depth}\left(M_{i_{1}} \otimes_{R} \ldots \otimes_{R} M_{i_{t}}\right) \geq 3$ whenever $1 \leq i_{1}<\ldots<i_{t} \leq n$. 
(2) $\sum_{i=1}^{n} \operatorname{depth}\left(M_{i}\right)=(n-1) d+\operatorname{depth}\left(M_{1} \otimes_{R} \ldots \otimes_{R} M_{n}\right) \geq(n-1) d+3$.

(3) If $n \geq d-2$ then at least one of the $M_{i}$ is free.

Example 3.2 shows that the inequalities in (3.4)(3) and (3.5)(3) cannot be improved, since $\mathrm{H}_{\mathrm{m}}^{1}\left(\otimes_{R}^{d-2} T\right)=\mathrm{H}_{\mathrm{m}}^{2}\left(\otimes_{R}^{d-3} T\right)=0$.

The next few results use the Syzygy Theorem [EG, (3.15)], which says that a non-free module having finite projective dimension and satisfying Serre's condition $\left(\mathrm{S}_{\mathrm{r}}\right)$ has rank at least $r$. The proof of the Syzygy Theorem uses "big Cohen-Macaulay modules", so we will need to restrict to regular local rings containing a field.

3.6. Proposition. Let $M$ be a vector bundle of rank 2 over a regular local ring $(R, \mathbf{m})$ containing a field. If $\mathrm{H}_{\mathbf{m}}^{2}\left(M \otimes_{R} N\right)=0$ for some nonzero vector bundle $N$, then $M^{*}$ is free.

Proof. If $d:=\operatorname{dim}(R) \leq 2$ then $M^{*}$ is free, being a second syzygy. Therefore we assume $d \geq 3$. By (3.3) we can assume both $M$ and $N$ are reflexive. Then $\operatorname{depth}(M)+\operatorname{depth}(N) \geq d+3$ by (2.4). Therefore $\operatorname{depth}(M) \geq 3$, so $M$ satisfies $\left(\mathrm{S}_{3}\right)$. Since the rank of $M$ is less than 3, the syzygy theorem [EG, (3.15)] implies that $M$ is free.

If we don't care which module turns out to be free we can do better:

3.7. Proposition. Let $M$ and $N$ be vector bundles over a regular local ring $(R, \mathbf{m})$ containing a field. Assume $\mathrm{H}_{\mathbf{m}}^{2}\left(M \otimes_{R} N\right)=0$. Then any of the following conditions implies that either $M^{*}$ or $N^{*}$ is free:

(1) $d:=\operatorname{dim}(R) \leq 4$.

(2) $\operatorname{rank}(M) \leq 3$ or $\operatorname{rank}(N) \leq 3$.

(3) $\operatorname{rank}(M)+\operatorname{rank}(N) \leq d+2$.

Proof. As in the proof of (3.6) we may assume that $d \geq 3$ and that $M$ and $N$ are reflexive. Let $g=\operatorname{depth}(M), h=\operatorname{depth}(N)$. By (2.2) we have $g+h \geq d+3$. We assume neither $M$ nor $N$ is free, that is, $g<d$ and $h<d$. Therefore $g \geq 4$ and $h \geq 4$. Also, by the Syzygy Theorem [EG, (3.15)], we have $\operatorname{rank}(M) \geq g$ and $\operatorname{rank}(N) \geq h$. This data obviously contradicts (1), (2) and (3).

Here is a expanded version of (3.7). The proof is essentially the same (but uses (3.5) instead of (2.4)) and is left to the reader.

3.8. Corollary. Let $M_{1}, \ldots, M_{n}$ be vector bundles over a regular local ring $(R, \mathbf{m})$ containing a field. Assume $\mathrm{H}_{\mathbf{m}}^{2}\left(M_{1} \otimes_{R} \ldots \otimes_{R} M_{n}\right)=0$, but none of $M_{1}^{*}, \ldots, M_{n}^{*}$ is free. Then

(1) $d:=\operatorname{dim}(R) \geq n+3$; and 
(2) For every positive integer $t \leq n$ and every sequence $1 \leq i_{1}<\ldots<i_{t} \leq n$ we have $\sum_{j=1}^{t} \operatorname{rank}\left(M_{i_{j}}\right) \geq(t-1)(d-1)+n+2$.

Perhaps it is worth spelling out these conditions for $n=3$ :

3.9. ExAmpLE. Let $M_{1}, M_{2}, M_{3}$ be non-free reflexive vector bundles over a regular local ring $R$ of dimension $d \geq 3$. Assume $R$ contains a field and that $\mathrm{H}_{\mathbf{m}}^{2}\left(M_{1} \otimes_{R} M_{2} \otimes_{R} M_{3}\right)=0$. Then $d:=\operatorname{dim}(R) \geq 6$, each $M_{i}$ has rank at least 5 , any two have ranks adding to at least $d+4$, and the sum of the ranks of all three is at least $2 d+3$.

The usefulness of (2.4) is some what limited by the assumption, in (2.4)(1), that both $M$ and $N$ have depth at least $r$. (Indeed, this requirement is what prevents us from extending the last few results to $\mathrm{H}_{\mathrm{m}}^{3}$ and beyond.) Unfortunately the assumption cannot be deleted, even if $N=R$ :

3.10. EXAMPLe. Let $R$ be a regular local ring of dimension $d$, and let $M=\mathbf{m}$ be the maximal ideal of $R$. Then $\mathrm{H}_{\mathrm{m}}^{i}(M)=0$ if and only if $i=1$ or $i=d$.

Proof. We know $\mathrm{H}_{\mathrm{m}}^{i}(M) \neq 0$ for $i=1$ and $i=d$, because these values are respectively the depth and the dimension. Since $R / M$ has finite length and the intermediate local cohomology modules vanish for $R$, they also vanish for $M$.

\section{Cohomology of $M \otimes_{R} M^{*}$.}

In this section we study the cohomology of $M \otimes_{R} M^{*}$, where $M$ is a vector bundle on the punctured spectrum of a regular local ring. We begin with the following application of the Hartshorne-Ogus Theorem [V, (3.3.16)] and (2.4):

4.1. THEOREM. Let $M$ be a vector bundle over a regular local ring $R$ of dimension $d \geq 2$.

(1) If $\mathrm{H}_{\mathbf{m}}^{1}\left(M \otimes_{R} M^{*}\right)=0$, then $M=F \oplus T$, where $F$ is free and $T$ has finite length.

(2) If $\mathrm{H}_{\mathbf{m}}^{2}\left(M \otimes_{R} M^{*}\right)=0$, then $M^{*}$ is free.

Proof. To prove (1) we may assume that $M$ is torsion-free by (3.3)(1). We have $\operatorname{depth}(M)+\operatorname{depth}\left(M^{*}\right) \geq d+2$ by (2.2). The Hartshorne-Ogus Theorem [V, (3.3.10)] now implies that $M$ is free. For (2), we may assume that $d \geq 3$ (since $M^{*}$ is a second syzygy and is therefore free if $d=2$ ). By (3.3) we have $\mathrm{H}_{\mathrm{m}}^{2}\left(M^{* *} \otimes_{R} M^{*}\right)=0$. Since both $M^{*}$ and $M^{* *}$ have depth at least 2, (2.2) implies that $\operatorname{depth}\left(M^{*}\right)+\operatorname{depth}\left(M^{* *}\right) \geq d+3$. Again, we apply [V, (3.3.16)] to conclude that $M^{*}$ is free. 
Theorem 4.1 (2) is one of the main theorems of Luk and Yau in [LY] when restricted to the case of vector bundles over projective space, as we shall discuss in the next section. See Theorem 5.2.

To get information on the higher local cohomology groups, we can sometimes use the following duality theorem. We let ()$^{\vee}$ denote the Matlis dual $\operatorname{Hom}_{R}(, E(k))$, where $E(k)$ is the injective hull of the residue field $k$.

4.2. THEOREM. Let $M$ and $N$ be vector bundles over a regular local ring $R$. Assume $d:=\operatorname{dim}(R) \geq 3$, and let $j$ be an integer with $2 \leq j \leq d-1$. Then $\left(\mathrm{H}_{\mathrm{m}}^{j}\left(M \otimes_{R} N\right)\right)^{\vee} \cong \mathrm{H}_{\mathrm{m}}^{d+1-j}\left(M^{*} \otimes_{R} N^{*}\right)$. In particular, $\mathrm{H}_{\mathrm{m}}^{j}\left(M \otimes_{R} N\right)=0$ if and only if $\mathrm{H}_{\mathrm{m}}^{d+1-j}\left(M^{*} \otimes_{R} N^{*}\right)=0$.

PROOF. There is a natural homomorphism $\Phi: M^{*} \otimes_{R} N^{*} \rightarrow\left(M \otimes_{R} N\right)^{*}$ taking $f \otimes g$ to the map $x \otimes y \mapsto f(x) \cdot g(y)$. If $M$ is free one checks easily (by choosing a basis) that $\Phi$ is an isomorphism. Therefore in general the kernel and cokernel of $\Phi$ have finite length. Since $d+1-j \geq 2$ it follows that

$$
\mathrm{H}_{\mathbf{m}}^{d+1-j}\left(M^{*} \otimes_{R} N^{*}\right) \cong \mathrm{H}_{\mathbf{m}}^{d+1-j}\left(\left(M \otimes_{R} N\right)^{*}\right) \text { for } i \geq 2 .
$$

It follows from Grothendieck's local duality theorem [BH, (3.3.8)] that

$$
\mathrm{H}_{\mathbf{m}}^{d+1-j}\left(\left(M \otimes_{R} N\right)^{*}\right) \cong\left(\mathrm{H}_{\mathbf{m}}^{j}\left(\left(M \otimes_{R} N\right)^{* *}\right)\right)^{\vee} .
$$

(See [BH,(3.5.15)].) Combining (4.2.1) and (4.2.2) with (3.3), we obtain the desired isomorphism.

4.3. Corollary. Let $M$ be a vector bundle over a d-dimensional regular local ring $(R, \mathbf{m})$. If $\mathrm{H}_{\mathrm{m}}^{d-1}\left(M \otimes_{R} M^{*}\right)=0$, then $M^{*}$ is free.

Proof. By (4.2) we have $\mathrm{H}_{\mathrm{m}}^{2}\left(M^{*} \otimes_{R} M^{* *}\right)=0$. Then (4.1) guarantees that $M^{* *}$ is free, whence so is $M^{*}$.

4.4. CoRollary. Let $M$ and $N$ be vector bundles over a regular local ring $(R, \mathbf{m})$ of dimension $d \geq 3$. Assume $\mathrm{H}_{\mathbf{m}}^{2}\left(M \otimes_{R} N\right)=\mathrm{H}_{\mathbf{m}}^{d-1}\left(M \otimes_{R} N\right)=0$. Then either $M^{*}$ or $N^{*}$ is free.

Proof. By (4.2) we have $\mathrm{H}_{\mathrm{m}}^{2}\left(M^{*} \otimes_{R} N^{*}\right)=0$, and $\mathrm{H}_{\mathrm{m}}^{2}\left(M^{* *} \otimes_{R} N^{* *}\right)=0$ by (3.3). Therefore $\operatorname{depth}\left(M^{*}\right)+\operatorname{depth}\left(N^{*}\right) \geq d+3$ and $\operatorname{depth}\left(M^{* *}\right)+$ $\operatorname{depth}\left(N^{* *}\right) \geq d+3$, by (2.2). It follows that either $\operatorname{depth}\left(M^{*}\right)+$ $\operatorname{depth}\left(M^{* *}\right) \geq d+3$ or $\operatorname{depth}\left(N^{*}\right)+\operatorname{depth}\left(N^{* *}\right) \geq d+3$. The HartshorneOgus Theorem [V, (3.3.16) implies that either $M^{*}$ or $N^{*}$ is free.

\section{Vector bundles on $\mathbf{P}^{n}$.}

One of the motivations for beginning work on this paper was our interest in the following theorem of Luk and Yau [LY, Theorem B]: 
5.1. THEOREM. Let $E$ be a holomorphic vector bundle on $\mathrm{P}_{\mathrm{C}}^{n}, n \geq 2$. Then $E$ is a direct sum of holomorphic line bundles iff $\mathrm{H}^{1}\left(\mathrm{P}_{\mathrm{C}}^{n},\left(E \otimes E^{*}\right)(k)\right)=0$ for all $k \in \mathbf{Z}$.

This theorem follows directly from our main results in Section 4. Even more, we can prove the following theorems:

5.2 TheOREM. Let $E$ be a vector bundle on $\mathrm{P}_{k}^{n}, k$ a field and $n \geq 2$. Then $E$ is a direct sum of line bundles iff $\mathrm{H}^{1}\left(\mathrm{P}_{k}^{n},\left(E \otimes E^{*}\right)(l)\right)=0$ for all $l \in \mathbf{Z}$.

5.3. THEOREM. Let $E$ be a rank 2 vector bundle on $\mathrm{P}_{k}^{n}, k$ a field and $n \geq 2$. If there exists a nonzero vector bundle $F$ such that $\mathrm{H}^{1}\left(\mathrm{P}_{k}^{n},(E \otimes F)(l)\right)=0$ for all $l \in \mathbf{Z}$, then $E$ is a direct sum of line bundles.

Theorems 5.1-5.3 are immediate corollaries of Theorem 2.4 and Proposition 3.6 once a translation is made. We review this translation here. Let $X=\mathrm{P}_{k}^{n}$, and let $E$ be a vector bundle on $X$. The module $M=\oplus_{l \geq 0} \mathrm{H}^{0}(X, E(l))$ is a graded reflexive module over the polynomial ring $S=k\left[X_{0}, \ldots, X_{n}\right]$. The sheaf associated with this module over $\operatorname{Proj}(S)=X$ is exactly $E$. The fact that $E$ is a vector bundle corresponds to the property that $M_{P}$ is free over $S_{P}$ for all $P \neq \mathbf{m}=\left(X_{0}, \ldots, X_{n}\right)$. To say $E$ is a direct sum of line bundles is equivalent to the statement that $M$ is a free $S$-module. We note:

5.4. Lemma. Let $S=k\left[X_{0}, \ldots, X_{n}\right]$ be a polynomial ring over a field $k$ with the usual grading. Let $M$ be a finitely generated graded reflexive $S$-module, and let $E$ be the associated sheaf over $X=\operatorname{Proj}(S)$. The following are equivalent.

(1) $M$ is a free $S$-module.

(2) $E$ is a direct sum of line bundles.

(3) $M_{\mathbf{m}}$ is a free $S_{\mathbf{m}}$-module, where $\mathbf{m}=\left(X_{0}, \ldots, X_{n}\right) S$.

Proof. The equivalence of the first two is discussed in the paragraph above. Clearly (1) implies (3), so it remains to see that (3) implies (1). The locus of primes $P$ in $\operatorname{Spec}(S)$ at which $M$ is not free is a closed set defined by a homogeneous ideal $I$. Assuming (3), $I \nsubseteq \mathbf{m}$. Since $I$ is homogeneous it follows that $I=S$, and $M$ is locally free, i.e. is a projective module. However, since $M$ is graded, it now follows that $M$ is a free $S$-module (cf. [E, (19.2)]).

We also recall the interpretation of sheaf cohomology in terms of local cohomology. The following is given as Theorem A4.1 in [E]:

5.5. Proposition. Let $R$ be a nonnegatively graded ring over a field $k=R_{0}$, and let $M$ be a graded $R$-module with associated sheaf $E$ over $X=\operatorname{Proj}(R)$. Let $\mathbf{m}$ be the irrelevant maximal ideal of all elements of positive 
degree. Then there are isomorphisms for every $i \geq 1$,

$$
\sum_{l} \mathrm{H}^{i}(X, E(l)) \cong \mathrm{H}_{\mathbf{m}}^{i+1}(M)
$$

where the sum extends over all integers.

Proof of (5.2). Let $S=k\left[X_{0}, \ldots, X_{n}\right]$ and let $M$ be the reflexive module corresponding to the vector bundle $E$. The condition on the global cohomology of $E$ in (5.2) translates by (5.5) to the condition that $\mathrm{H}_{\mathbf{m}}^{2}\left(M \otimes_{S} M^{*}\right)=0$. If this condition holds, we localize at $\mathbf{m}=\left(X_{0}, \ldots, X_{n}\right) S$ and let $T=S_{\mathrm{m}}$. We then have that $M_{\mathrm{m}}$ is a vector bundle in the sense of (3.0) and $\mathrm{H}_{\mathbf{m}}^{2}\left(M_{\mathbf{m}} \otimes_{T} M_{\mathrm{m}}^{*}\right)=0$. Theorem 4.1 implies that $M_{\mathbf{m}}$ is free over $S_{\mathrm{m}}$, and $E$ is a direct sum of line bundles by (5.4). The converse is trivial since $\operatorname{dim}(S)=n+1>2$.

Proof of (5.3). Let $S=k\left[X_{0}, \ldots, X_{n}\right]$ and let $M$ be the reflexive module corresponding to the rank two vector bundle $E$, and let $N$ be the $S$-module corresponding to the vector bundle $F$. The condition on the global cohomology of $E \otimes F$ in (5.3) translates by (5.5) to the condition that $\mathrm{H}_{\mathbf{m}}^{2}\left(M \otimes_{S} N\right)=0$. Localize at $\mathbf{m}=\left(X_{0}, \ldots, X_{n}\right) S$ and let $T=S_{\mathbf{m}}$. We then have that $M_{\mathrm{m}}$ and $N_{\mathrm{m}}$ are vector bundles in the sense of (3.0) and that $\mathrm{H}_{\mathbf{m}}^{2}\left(M_{\mathbf{m}} \otimes_{T} N_{\mathbf{m}}\right)=0$. From Proposition 3.6 it follows that $M_{\mathbf{m}}$ is free over $S_{\mathrm{m}}$, and then from (5.4) that $E$ is a direct sum of line bundles.

\section{A Rigidity Theorem.}

In this section we prove a new rigidity theorem for the depths of Tors over unramified regular local rings. As in Auslander's proof of the original theorem on the rigidity of Tor, we reduce our main statement to a statement concerning Koszul homology. We begin with two elementary lemmas. We use $\lambda(\quad)$ to denote the length of a module.

6.1. Lemma. Let $(R, \mathbf{m})$ be a Noetherian local ring, and let $M$ be a finitely generated R-module. Fix $x \in R$.

(1) $\lambda\left(\operatorname{Ann}_{M}(x)\right)<\infty$ implies $\lambda\left(\operatorname{Ann}_{M}\left(x^{n}\right)\right)<\infty$ for all $n \geq 1$.

(2) $\lambda(M / x M)<\infty$ implies $\lambda\left(M / x^{n} M\right)<\infty$ for all $n \geq 1$.

Proof. Let $f: M \rightarrow M$ be multiplication by $x . \lambda\left(\operatorname{Ann}_{M}(x)\right)<\infty$ iff $f_{P}$ is injective for every prime $P \neq \mathbf{m}$ iff $f_{P}^{n}$ is injective for all $P \neq \mathbf{m}$ iff $\lambda\left(\operatorname{Ann}_{M}\left(x^{n}\right)\right)<\infty$.

Similarly $\lambda(M / x M)<\infty$ iff $f_{P}$ is surjective for every prime $P \neq \mathbf{m}$ iff $f_{P}^{n}$ is surjective for all $P \neq \mathbf{m}$ iff $\lambda\left(M / x^{n} M\right)<\infty$.

6.2. Lemma. Let $(R, \mathrm{~m})$ be a Noetherian local ring, and let $M$ be a 
finitely generated R-module. Fix $x \in R$. If $0<\lambda\left(\operatorname{Ann}_{M}(x)\right)<\infty$ then $\operatorname{depth}(M / x M)=0$.

Proof. $\operatorname{Ann}_{M}(x) \subseteq \operatorname{Ann}_{M}\left(x^{2}\right) \subseteq \ldots \subseteq \operatorname{Ann}_{M}\left(x^{n}\right) \subseteq \ldots$, so we may choose $n$ so that this chain is stable. Set $L$ equal to the stable submodule. By Lemma $6.1 L$ has finite length. Suppose that $\operatorname{depth}(M / x M)>0$. The image of $L$ in this module must then be 0 , so that $L \subseteq x M$. Given $z \in L$ write $z=x y$. Then $z x^{n}=0$ implies $y x^{n+1}=0$ implies $y \in L$ and so $L=x L$. Nakayma's lemma applies to give $L=0$, a contradiction.

6.3. TheOREM. Let $(R, \mathbf{m})$ be a Noetherian local ring, and let $M$ be a finitely generated R-module. Fix $x_{1}, \ldots, x_{n} \in \mathbf{m}$. Assume that the Koszul homology $\mathrm{H}_{1}\left(x_{1}, \ldots, x_{n} ; M\right)$ is a nonzero module of finite length. Then depth $\mathrm{H}_{0}\left(x_{1}, \ldots, x_{n} ; M\right)=0$.

Proof. The long exact sequence on Koszul homology [Ma, p. 128] induces a short exact sequence

$$
0 \rightarrow K / x_{n} K \rightarrow \mathrm{H}_{1}\left(x_{1}, \ldots, x_{n} ; M\right) \rightarrow \operatorname{Ann}_{N}\left(x_{n}\right) \rightarrow 0
$$

where $K=\mathrm{H}_{1}\left(x_{1}, \ldots, x_{n-1} ; M\right)$ and $N=\mathrm{H}_{0}\left(x_{1}, \ldots, x_{n-1} ; M\right)$.

Assume that depth $\mathrm{H}_{0}\left(x_{1}, \ldots, x_{n} ; M\right)>0$.

6.3.2. Claim. $\operatorname{Ann}_{N}\left(x_{n}\right)=0$. If not then $0 \neq \lambda\left(\operatorname{Ann}_{N}\left(x_{n}\right)\right)<\infty$, which follows from (6.3.1) since $\mathrm{H}_{1}\left(x_{1}, \ldots, x_{n} ; M\right)$ has finite length. Lemma 6.2 then gives that $N / x_{n} N \cong \mathrm{H}_{0}\left(x_{1}, \ldots, x_{n} ; M\right)$ has depth 0 , contradicting our assumption. Hence $\operatorname{Ann}_{N}\left(x_{n}\right)=0$ and $K / x_{n} K \cong \mathrm{H}_{1}\left(x_{1}, \ldots, x_{n} ; M\right)$.

Now replace $x_{n}$ by $x_{n}^{k}$ in (6.3.1). Since $x_{n}$ is not a zero-divisor on $N, x_{n}^{k}$ will also be a non-zero-divisor and we obtain isomorphisms,

$$
K / x_{n}^{k} K \cong \mathrm{H}_{1}\left(x_{1}, \ldots, x_{n}^{k} ; M\right)
$$

for all $k \geq 1$. Moreover by Lemma $6.1, K / x_{n}^{k} K$ must have finite length, and consequently so does $\mathrm{H}_{1}\left(x_{1}, \ldots, x_{n}^{k} ; M\right)$. In addition, depth $\mathrm{H}_{0}\left(x_{1}, \ldots, x_{n}^{k} ; M\right)=$ depth $N / x_{n}^{k} N=$ depth $N / x_{n} N=$ depth $\mathrm{H}_{0}\left(x_{1}, \ldots, x_{n} ; M\right)>0$. It follows that if the elements $x_{1}, \ldots, x_{n}$ give a counterexample to the statement of the theorem, then so do $x_{1}, \ldots, x_{n}^{k}$ for every $k \geq 1$. In addition every permutation of elements $x_{1}, \ldots, x_{n}$ giving a contradiction to the statement of the theorem also gives a counterexample, as the Koszul homology does not change after rearranging the elements $x_{i}$. By iterating the argument, we can conclude that for all $k_{i} \geq 1$,

$$
\lambda\left(\mathrm{H}_{1}\left(x_{1}^{k_{1}}, \ldots, x_{n}^{k_{n}} ; M\right)\right)<\infty \text { and depth } \mathrm{H}_{1}\left(\mathrm{x}_{1}^{\mathrm{k}_{1}}, \ldots, \mathrm{x}_{\mathrm{n}}^{\mathrm{k}_{\mathrm{n}}} ; \mathbf{M}\right)>0 .
$$

By Claim 6.3.2, $x_{i}^{k_{1}}$ is a non-zero-divisor on the module $M /\left(x_{1}^{k_{1}}, \ldots, x_{i-1}^{k_{t-1}}, x_{i+1}^{k_{t+1}}, \ldots, x_{n}^{k_{n}}\right) M$. We now claim by induction on $s$ that 
$x_{1}, \ldots, x_{s}$ form a regular sequence on $M$. Suppose we have shown this for some $s, 0 \leq s \leq n-1$. After rearrangement we know that $x_{s+1}$ is a non-zerodivisor on the module $M /\left(x_{1}, \ldots, x_{s}, x_{s+2}^{k_{s+2}}, \ldots, x_{n}^{k_{n}}\right) M$ for every $k_{i} \geq 1$. Letting the $k_{i}$ go to infinity gives that $x_{s+1}$ is a non-zero-divisor on $M /\left(x_{1}, \ldots, x_{s}\right) M$, which proves the claim. But $x_{1}, \ldots, x_{n}$ a regular sequence on $M$ proves that $\mathrm{H}_{1}\left(x_{1}, \ldots, x_{n} ; M\right)=0$. This contradiction proves the theorem.

6.4. TheOREM. Let $M$ and $N$ be finitely generated modules over the regular local ring $(R, \mathbf{m})$. Suppose, for some positive integer $n$, that $\operatorname{Tor}_{n}^{R}(M, N)$ is a nonzero module of finite length. If $n=2$ assume that either $M$ or $N$ has positive depth. Assume $R$ is either equicharacteristic or unramified; and if $n=1$ and $R$ is not equicharacteristic, assume that $\operatorname{char}(R / \mathbf{m})$ is a non-zero-divisor either on $M$ or on $N$. Then $\operatorname{Tor}_{n-1}^{R}(M, N)$ has depth 0 .

Proof. Suppose $n=1$. We may assume that $R$ is complete, so that $R \cong k\left[\left[X_{1}, \ldots, X_{r}\right]\right]$, where $k$ is either a field or a discrete rank-one valuation ring. In either case, our hypotheses imply that $\operatorname{Tor}_{i}^{R}(M, N) \cong$ $\mathrm{H}_{i}\left(x_{1}, x_{r} ; M \hat{\otimes}_{k} N\right.$ ), where $M \hat{\otimes}_{k} N$ is the complete tensor product (which is a finitely generated $k\left[\left[X_{1}, \ldots, X_{r}, Y_{1}, \ldots, Y_{r}\right]\right]$-module) and where $x_{j}=X_{j}-Y_{j}$. (See [S, Chap. V, Part, B, -p2] and [A1, pp. 635-636].) The desired result nowfollows from (6.3).

Suppose next that $n=2$ and $N$ (say) has positive depth. Choose an exact sequence

$$
0 \rightarrow M_{1} \rightarrow F \rightarrow M \rightarrow 0
$$

with $F$ free. Then $\operatorname{Tor}_{1}^{R}\left(M_{1}, N\right) \cong \operatorname{Tor}_{2}^{R}(M, N)$, which by assumption is a nonzero module of finite length. Since $M_{1}$ is torsion-free, the case $n=1 \mathrm{im}$ plies that $\operatorname{depth}\left(M_{1} \otimes_{R} N\right)=0$. Now tensor (6.4.1) with $N$, getting an exact sequence

$$
0 \rightarrow \operatorname{Tor}_{1}^{R}(M, N) \rightarrow M_{1} \otimes_{R} N \stackrel{\alpha}{\rightarrow} F \otimes_{R} N \rightarrow M \otimes_{R} N \rightarrow 0 .
$$

Since $N$ has positive depth, the image $C$ of $\alpha$ also has positive depth. The exact sequence

$$
0 \rightarrow \operatorname{Tor}_{1}^{R}(M, N) \rightarrow M_{1} \otimes_{R} N \rightarrow C \rightarrow 0
$$

and the fact that $\operatorname{depth}\left(M_{1} \otimes_{R} N\right)=0$ now imply that $\operatorname{depth}\left(\operatorname{Tor}_{1}^{R}(M, N)\right)=0$ as desired.

Finally, assume that $n \geq 3$. Let $N^{\prime}$ be the $(n-2)^{\text {nd }}$ syzygy of $N$. Then $\operatorname{Tor}_{2}^{R}\left(M, N^{\prime}\right) \cong \operatorname{Tor}_{n}^{R}(M, N)$, and $\operatorname{since} \operatorname{depth}\left(N^{\prime}\right)>0$ the case $n=2$ implies that $\operatorname{Tor}_{1}^{R}\left(M, N^{\prime}\right)$ has depth 0 . Since $\operatorname{Tor}_{1}^{R}\left(M, N^{\prime}\right) \cong \operatorname{Tor}_{n-1}^{R}(M, N)$ we are done. 


\section{REFERENCES}

[A1] M. Auslander, Modules over unramified regular local rings, Illinois J. Math. 5 (1961), 631-647.

[A2] M. Auslander, On the purity of the branch locus, Amer. J. Math. 84 (1962), 116-125.

[A3] M. Auslander, Modules over unramified regular local rings, Proc. International Congress of Mathematics in Stockholm 1962, Uppsala, Almqvist and Wiksells (1963), 230-234.

[AB1] M. Auslander and D. Buchsbaum, Invariant factors and two criteria for projectivity of modules, Trans. Amer. Math. Soc. 104 (1962), 516-522.

[AB2] M. Auslander and D. Buchsbaum, On ramification theory in noetherian rings, Amer. J. Math. 81 (1959), 749-764.

[AG] M. Auslander and O. Goldman, Maximal orders, Trans. Amer. Math. Soc. 97 (1960), $1-24$.

[BH] W. Bruns and J. Herzog, Cohen-Macaulay Rings, Cambridge Stud. in Adv. Mathematics 39 (1993).

[C] P. Constapel, Length of Tor and torsion in tensor products, Thesis, Northwestern University (1995).

[DHM] S. Dutta, M. Hochster, J. McLaughlin, Modules of finite projective dimension with negative intersection multiplicities, Invent. Math. 79 (1985) 253-291.

[E] D. Eisenbud, Commutative Algebra with a View Toward Algebraic Geometry, Graduate Texts in Math. 150 (1995).

[EG] E. G. Evans and P. Griffith, Syzygies, London Math. Soc. Notes Ser. 106 (1985).

[HW] C. Huneke and R. Wiegand, Tensor products of modules and the rigidity of Tor, Math. Ann. 299 (1994), 439-476.

[L] S. Lichtenbaum, On the vanishing of Tor in regular local rings, Illinois J. Math. 10 (1966) 220-226.

[LY] H. S. Luk and S. S.-T. Yau, Cohomology and splitting criterion for holomorphic vector bundles on $\mathbf{C P}^{n}$ Math. Nachr. 161 (1993), 233-238.

[Ma] H. Matsumura, Commutative Ring Theory Cambridge Stud. Adva. Math. 8 (1990).

[Mu] M. P. Murthy, Modules over regular local rings, Illinois J. Math. 7 (1963), 558-565.

[S] J. P. Serre, Algèbre Locale: Multiplicités, Springer Lecture Notes 11 (1965).

[V] W. Vasconcelos, Arithmetic of Blowup Algebras, London Math. Soc. Lecture Notes 195 (1994).

DEPARTMENT OF MATHEMATICS

PURDUE UNIVERSITY

WEST LAFAYETTE, IN 47907

USA

E-mail address·huneke@math.purdue.edu
DEPARTMENT OF MATHEMATICS

UNIVERSITY OF NEBRASKA

LINCOLN, NE 68588-0323

USA

E-mail address: rwiegand $@$ unl.edu 